Annls Limnol. 10 (2) $1974: 121-130$.

\title{
DIPTẼRES TORRENTICOLES PEU CONNUS : \\ II. - LES ATHERICIDAE (NYMPHES) DU SUD DE LA FRANCE
}

\author{
[Brachycera, Orthorrhapha]
}

\author{
par A. G. B. Thomas'
}

Cette note contient une description comparée et des figures des nymphes des trois espèces Atherix ibis (Fabricius), Atherix marginata (Fabricius) et Atrichops crassipes (Meigen). La disposition des stigmates est du type péripneustique. Le stade nymphal d'Atrichops crassipes était inconnu jusqu'ici.

\section{Poorly known torrential Diptera : \\ II. - Pupae of Athericidae (Brachycera, Orthorrhapha) from the South of France.}

The pupae of three species : Atherix ibis (Fab.), Atherix marginata (Fab.) and Atrichops crassipes (Meigen) are compared and illustrated. The arrangement of the stigmata is of the peripneustic type. The pupae of Atrichops crassipes is described for the first time.

La présente étude fait suite à une note concernant les imagos et les larves des mêmes espèces (Thomas 1974).

Les nymphes des Athericidae sont encore plus mal connues que les larves. Jusqu'ici, un seul travail détaillé a élé consacré aux nymphes de ces Diptères, celui de Nagatomi (1961) sur cinq des espèces japonaises. A ma connaissance, aucun manuel traitant de la biologie des eaux courantes ne mentionne le stade nymphal des Athericidae, aucune faune relative aux insectes aquatiques ne permet d'identification spécifique.

Les exuvies nymphales des Athericidae sont cependant souvent abondantes aux abords immédiats d'un grand nombre de cours d'eau à certaines périodes de l'année. Leurs récoltes, particulièrement aisées pour deux espèces, apportent d'autre part des précisions intéressantes sur la biologie et la répartition de celles-ci.

1. Laboratoire d'Hydrobiologie, Université Paul-Sabatier, 118, route de Narbonne, 31077 Toulouse Cedex, France. 


\section{Caractères communs aux trois espèces.}

Une partie de la terminologie utilisée est empruntée à Brauns (1954) et Séguy (1967).

La silhouette de la nymphe est le plus souvent à peu près rectiligne, parfois plus ou moins incurvée, en général sur la face dorsale. Par rapport à la larve, la perte de longueur est considérable, de l'ordre de 35 à $40 \%$ (par exemple, une larve d'A. marginata de $14 \mathrm{~mm}$ a été à l'origine d'une nymphe de $8,5 \mathrm{~mm}$ ).

TÊTE.

Céphalothèque volumineuse, de forme assez hémisphérique, en grande partie constituée par deux ophthalmothèques ( $p l$. I, fig. 1) proéminentes, séparées de la notothèque par un sillon transversal très accusé. Ce sillon passe sous les cornes prothoraciques et longe latéralement la partie proximale apparente des pédothèques (fig. 1 et 2 ). Les cératothèques ( $f \mathrm{~g} g .1$ et 3 ) sont petites et coniques, très écartées l'une de l'autre et situées assez près du bord latéral de la céphalothèque. Elles sont en grande partie séparées de cette dernière et ne recouvrent les antennes de l'imago que sur une longueur très réduite (tout au plus le dernier cinquième de l'arista chez $A$. marginata). Stomatothèque (fig. 3 ) de grandes dimensions, laissant deviner les contours des labelles de l'imago. Etuis des palpes maxillaires disposés transversalement, à extrémité distale aiguë et située exactement sous la cératothèque correspondante.

\section{Thorax.}

Cornes prothoraciques latéro-dorsales volumineuses, courtes et épaisses, plus larges que hautes.

Mésothorax volumineux, convexe, dépassant en hauteur la tête et l'abdomen. En vue latérale, ptérothèques ( $f i g .1$ ) inclinées à $45^{\circ}$ environ vers la partie postérieure du corps. Ventralement, l'extrémité des pédothèques ne dépasse guère les ptérothèques vers l'arrière.

Métathorax réduit mais toujours bien visible : sa longueur au niveau du plan de symétrie est égale à la plus grande dimension des cornes prothoraciques ou assez comparable.

\section{Abdomen.}

Il est formé de 8 segments apparents; le sternite du premier disparaît en grande partie sous les ptérothèques. Les segments 1 à 7 inclus sont de morphologie voisine et portent des ornementations assez comparables : dorsalement, sur chaque plaque tergale, 4 prolongements longs et grêles régulièrement espacés, latéralement 2 prolongements du même type (avec quelques variations pour le 
premier segment) et ventralement, sur chaque plaque sternale, 6 épines plus ou moins longues (absentes sur le premier segment). Le dernier segment porte une couronne plus ou moins régulière de fortes épines et deux prolongements terminaux coniques. Largeur maximale de l'abdomen en général au niveau des segments 3 ou 4 .

Chez Atherix ibis et $A$. marginata, on peut fréquemment observer à l'œil nu une nette alternance de zones brun foncé et brun jaunâtre qui confère à l'abdomen un aspect annelé.

Les segments abdominaux 1 à 7 portent chacun une paire de petits stigmates, bien visibles sur les exuvies nymphales des deux espèces Atherix ibis et $A$. marginata. Ces stigmates sont en relation avec un système de trachées apparemment simples. Cette disposition, voisine de celle rencontrée chez le genre Chrysopilus, est donc de type péripneustique et non propneustique comme l'indique Nagatomi (1961).

La déhiscence est longitudinale et médiane depuis la limite postérieure du mésonotum (l'enveloppe du métanotum ne se déchire pas) jusqu'au milieu environ de la courbure de la céphalothèque. A ce niveau, la déchirure se poursuit presque jusqu'au bord latéral de la céphalothèque selon deux lignes de déhiscence antérieures, à peu près orthogonales, en direction des pédothèques. Ces deux lignes passent juste au-dessus des cératothèques.

Atherix ibis (Fabricius, 1798) : $P l$. I, fig. 1 à 9 .

Cette nymphe n'a jamais été étudiée en Europe. La seule description antérieure se rapporte à la sous-espèce japonica dans le travail détaillé de Nagatomi (1961). Contrairement à ce qu'indique cet auteur, Dufour (1862) n'a ni décrit ni même mentionné la nymphe d'A. ibis.

Longueur totale du ô : 10 à $13 \mathrm{~mm}$, de la ㅇ : 12 à $15 \mathrm{~mm}$.

La coloration varie de jaune foncé à brun selon les spécimens, le stade de dóveloppement et les différentes régions de chaque nymphe. La coloration des exuvies est évidemment plus claire et moins contrastée. Certaines exuvies de grande taille peuvent être très foncées d'une extrémité à l'autre. D'une façon générale, pleures et sternites sont plus claires que les tergites fréquemment d'un brun soutenu.

\section{TÊTE.}

Ophthalmothèques très volumineuses, plus proéminentes que chez $A$. marginata et plus développées chez le ô que chez la \&. Cératothèques coniques, environ doux fois plus longues que larges à la base, inclinées de 30 à $45^{\circ}$ par rapport au plan de symétrie. Une paire de soies insérées chacune sur un petit mamelon à 
l'extrémité antérieure du corps et dirigées vers l'avant; une autre paire de soies, plus petites, insérées au-dessus des cératothèques et dirigées perpendiculairement aux précédentes.

\section{Thorax.}

Surface supérieure des cornes prothoraciques nettement bilobée, contour antérieur en forme de «3» (fig. 4). Soies du mésonotum fines et malaisées à observer ; de chaque côté, on en distingue une en arrière de la corne prothoracique au-dessus du bord antérieur de la ptérothèque et trois autres disposées en triangle à peu près équilatéral ( $f i g .2)$.

\section{Aвdomen.}

Prolongements dorsaux régulièrement espacés (fig. 2) ; prolongements latéraux de plus fort diamètre que les précédents et inclinés vers l'arrière. Stigmates en général alignés avec les bases des prolongements latéraux et situés en avant de ceux-ci (fig. 1). De chaque côté du premier segment, un seul prolongement latéral, de taille réduite, contre le bord postérieur de la ptérothèque, vers son milieu. Tous ces prolongements sont fortement barbelés (fig. 6). Ventralement, les segments 2 à 7 inclus portent chacun 6 épines coniques, dirigées vers l'arrière, non barbelées, deux à trois fois plus longues que larges à la base (fig. 5). Ces épines sont assez régulièrement espacées, l'intervalle entre les deux centrales étant souvent le plus faible des cinq.

Le segment anal porte une couronne de 6 longues et fortes épines aiguës à bord antérieur à peu près rectiligne. Ces épines sont régulièrement espacées et de longueur assez voisine (fig. 7 ). A l'extrémité du corps, deux forts prolongements coniques longitudinaux, de taille proportionnellement supérieure chez le $\hat{o}$ ( $f \mathrm{~g} g .8$ et 9 ).

Matériel examiné : 94 nymphes ou exuvies nymphales.

- Hautes-Pyrénées : $50 \mathrm{n}$.

Le ruisseau de Péguère à $1400 \mathrm{~m}$; la Neste de Couplan à 1380 et $1210 \mathrm{~m}$;

le r. de Lassas à $1250 \mathrm{~m}$.

- Haute-Garonne : $5 \mathrm{n}$.

L'Escalette à $410 \mathrm{~m}$.

- Tarn : 22 n. (3 imagos $\sigma$ et 2 i. ? obtenues par élevage).

Le r. de Laudot à $480 \mathrm{~m}$.

— Lozère : 14 n. (4 i. $\delta, 1$ i. 9 obtenues par élevage).

La Truyère à 1210 et $930 \mathrm{~m}$.

- Var: 1 n. ( 1 i. o obtenue par élevage).

La Gordolasque à $1540 \mathrm{~m}$ (F. Vaillant leg.).

- Isère : 2 n.

Le Furon à $980 \mathrm{~m}$. 

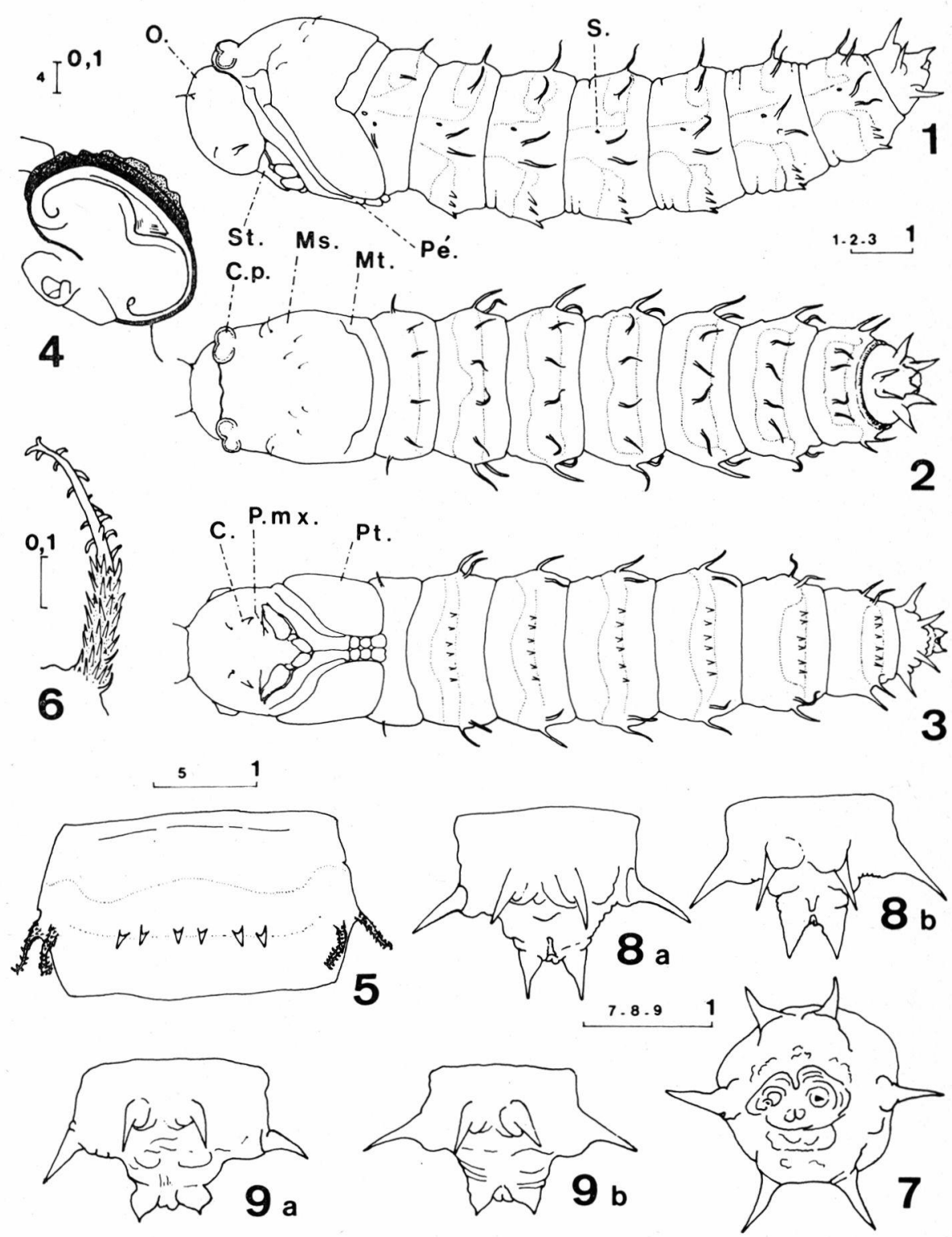

Planche I : fig. 1 à 9 (nymphe d'A. ibis). Échelle en mm.

FIG. 1 : vue latérale gauche; Fig. 2 : vue dorsale; Fig. 3 : vue ventrale ; FIg. 4: corne prothoracique, vue dorsale; FIG. 5: 4 e sternite abdominal; Fig. 6 : prolongement dorso-latéral du 4 e tergite ; Fig. 7 : segment anal, face postérieure ; FIG. 8 et 9 : appendices terminaux en vue dorsale, ${ }^{*}$ (fig. 8 ), ㅇ (fig. 9).

C. : cératothèque ; C.p. : corne prothoracique ; Ms : mésothorax ; Mt : métathorax ; O. : ophthalmothèque ; P. mx. : étui d'un palpe maxillaire ; Pé. : pédothèque ; $\mathrm{Pt}$. : ptérothèque ; $\mathrm{St}$. : stomatothèque ; $\mathrm{S}$. : stigmate abdominal. 
Atherix marginata (Fabricius, 1781) : Pl. II, fig. 10 à 16.

La nymphe de cette espèce n'a jamais été étudiée. Seul, Pomeisl (1953) en donne une figure sommaire, sans légende ni description.

Longueur totale : de 8,5 à $13 \mathrm{~mm}$.

La coloration rappelle celle d'A. ibis mais le mésothorax porte une ligne longitudinale médio-dorsale d'un brun soutenu, s'élargissant vers le métathorax. Cette ligne est entourée de deux bandes jaunes, concaves vers l'extéricur, qui s'étendent en avant jusqu'aux cornes prothoraciques incluses et qui régressent vers la fin de la nymphose ; latéralement, la coloration est brun foncé, s'estompant vent ralement.

TÊTE.

Cératothèques (fig. 10 et 12 ) courtes, coniques, de longueur rarement supérieure à une fois et demie leur largeur à la basc, peu inclinées par rapport au plan de symétrie $\left(0\right.$ à $30^{\circ}$ en général). Soies plus petites que celles d'A. ibis, de localisation comparable.

Thorax.

Surface supérieure des cornes prothoraciques fréquemment unilobée ou formée de deux lobes peu marqués. Soies de petite taille et peu visibles ( $f i g .10$ et 11 ) : trois sur la partie centrale de la notothèque (une postéricure proche de la ligne de déhiscence, deux antérieures groupées et plus éloignées de celle-ci), une sur le petit sclérite précédant immédiatement la ptérothèque.

\section{AbDomen.}

Prolongements dorsaux centraux (fig. 11) de taille réduite sur le second segment et surtout sur le premier. Du $5^{\mathrm{e}}$ au $7^{\mathrm{e}}$ tergite inclus, il $\mathbf{y}$ a intercalation entre les prolongements dorsaux et latéraux de tubercules épineux de volume croissant : un tubercule de chaque côté entre prolongements central et latéral, deux entre les prolongements centraux ( $f i g .15)$. Ces derniers sont eux-mêmes transformés, sur le $7^{\circ}$ tergite, en fortes épines assez semblables à celles de la couronne anale. Latéralement, le premier segment porte, comme les suivants, deux prolongements (fig. 10). Les stigmates se trouvent en général au-dessus du prolongement latéral antérieur. Tous ces prolongements sont proportionnellement plus longs, plus grêles et d'aspect encore plus nettement barbelé que chez $A$. ibis (fig. 14). Epines abdominales du second au $7^{\circ}$ segment inclus alignées en deux groupes de trois, l'intervalle médian nettement supérieur aux autres (fig. 12 et 13 ). Ces épines, grêles dès le cône basal, sont du même type que les prolongements dorsaux 

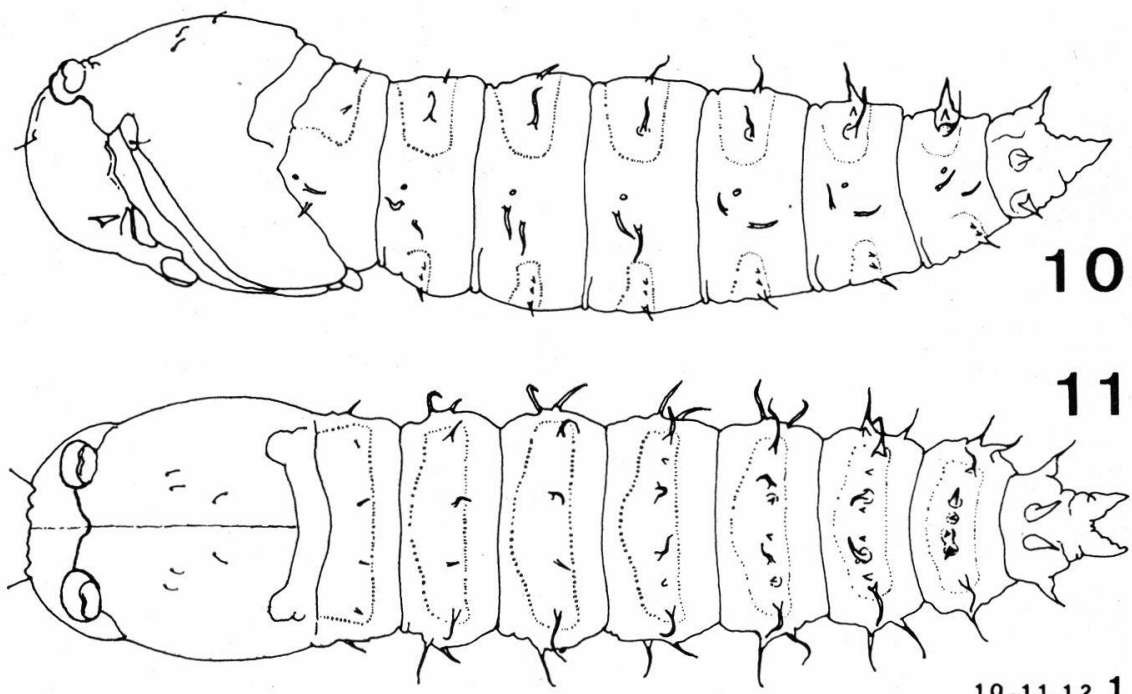

10.11 .121
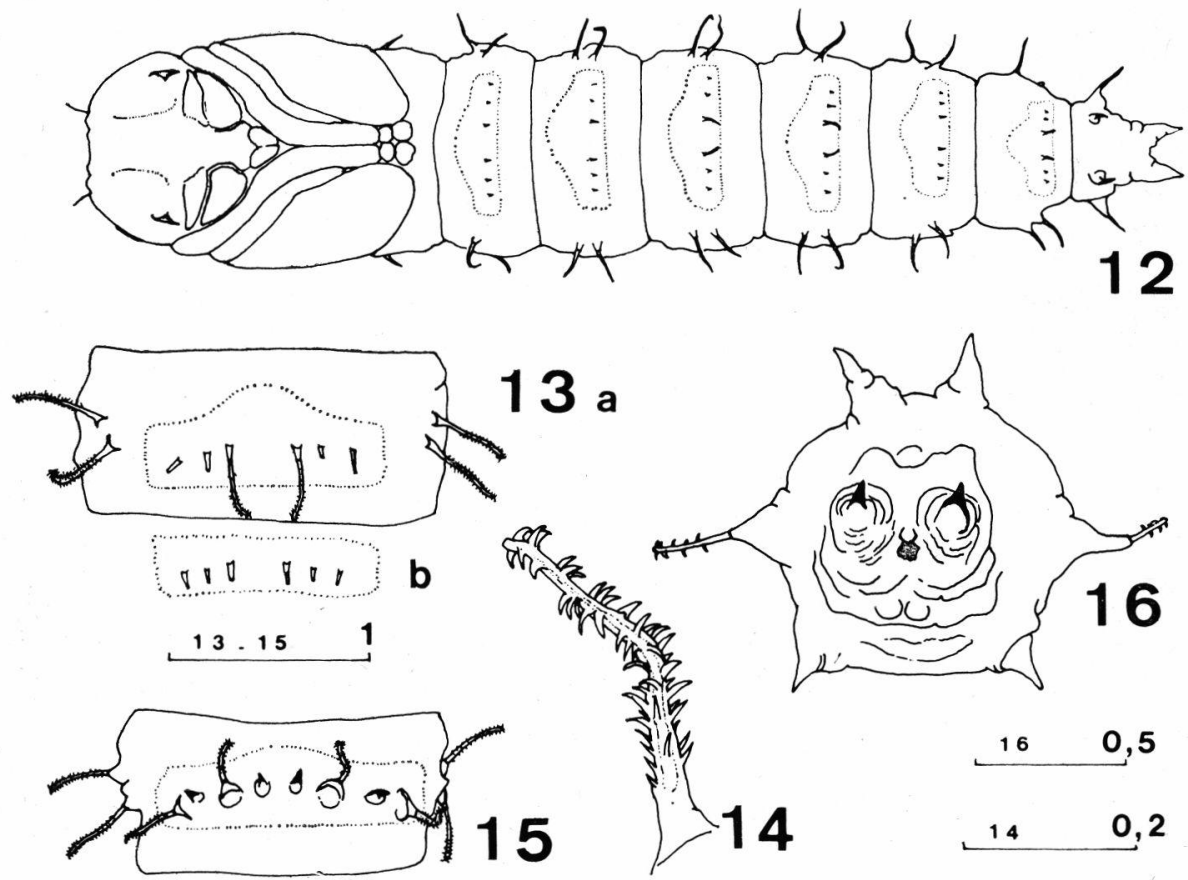

Planche II : fig. 10 à 16 (nymphe d'A. marginata). Échelle en mm.

FIg. 10 : vue latérale gauche; Fig. 11 : vue dorsale; Fig. 12 : vue ventrale; Fig. $13 \mathrm{a}: 4 \mathrm{e}$ sternite abdominal; Fig. $13 \mathrm{~b}:$ autre aspect fréquent des épines sternales ; Fig. 14 : prolongement dorso-latéral du 4 e tergite ; Fig. 15 : 6 ${ }^{\text {e }}$ tergite abdominal; Fig. 16 : segment anal, face postérieure. 
mais qui seraient plus ou moins atrophiés et fréquemment brisés. La paire centrale, incurvée symétriquement vers l'intérieur, est la plus longue et demeure souvent intacte.

Les épines de la couronne anale (fig. 16) sont plus courtes et moins aiguës que chez $A$. ibis. Leur bord antérieur est en général nettement moins rectiligne. Les deux épines inférieures sont presque parallèles, de taille réduite et fortement écartées à la base. L'épine latérale porte un prolongement barbelé semblable aux appendices tergaux. Les deux prolongements terminaux sont beaucoup plus volumineux que chez $A$. ibis et assez semblables pour les deux sexes.

A l'état nymphal comme aux états imaginal et larvaire, Suragina kodamai Nagatomi paraît être, de toutes les espèces décrites, la plus proche d'A. marginata.

Matériel examiné : 102 nymphes ou exuvies nymphales.

- Hautes-Pyrénées : 62 n. (2 i. đ" 2 i. \& obtenues par élevage).

La Neste de Couplan à $1210 \mathrm{~m}$; le r. d'Ardengost à $1150 \mathrm{~m}$; le Lavedan à 1080 et $900 \mathrm{~m}$; la Mousquère à 1050 et $950 \mathrm{~m}$; le r. de Beyrède à $1000 \mathrm{~m}$; le r. de Lassas à $980 \mathrm{~m}$.

- Ariège : $3 \mathrm{n}$.

Le Volp à $430 \mathrm{~m}$; un ruisselet affluent de l'Arize à $300 \mathrm{~m}$.

- Haute-Garonne : 11 n. ( 2 i. $\$$ obtenues par élevage).

L'Escalette à $410 \mathrm{~m}$; la Louge à $200 \mathrm{~m}$; la Garonne à $140 \mathrm{~m}$ (en 1967).

- Aude : $7 \mathrm{n}$.

L'Alzeau à $490 \mathrm{~m}$; le Tenten à 240 et $160 \mathrm{~m}$.

- Tarn : 17 n. (1 i. ơ, 2 i. $q$ obtenues par élevage).

Le Laudot à $480 \mathrm{~m}$,

一 Lozère : $1 \mathrm{n}$.

Le Lot à $1000 \mathrm{~m}$.

一 Isère : $1 \mathbf{n}$.

Le Furon à $980 \mathrm{~m}$.

Atrichops crassipes (Meigen, 1820) : $P l$. III, fig. 17 à 22.

La nymphe de cette espèce était inconnue jusqu'ici.

Longueur : $6 \mathrm{~mm}($ ( ) $)$.

Coloration : jaune grisâtre (pleures) à brun clair (mésothorax).

TÊTE.

Les deux paires de soies sont proportionnellement beaucoup plus développées que chez les deux autres espèces. Leur localisation est assez comparable mais l'insertion de chaque soie antérieure est protégée par un fort bourrelet dorsal (fig. 17). La ligne de déhiscence passe, en avant des cornes prothoraciques, par une protubérance médiane bien plus saillante que chez les deux Atherix. Les cératothèques (fig. 17 et 19) sont beaucoup plus longues (au moins trois fois plus longues que larges à la base) et leur extrémité 

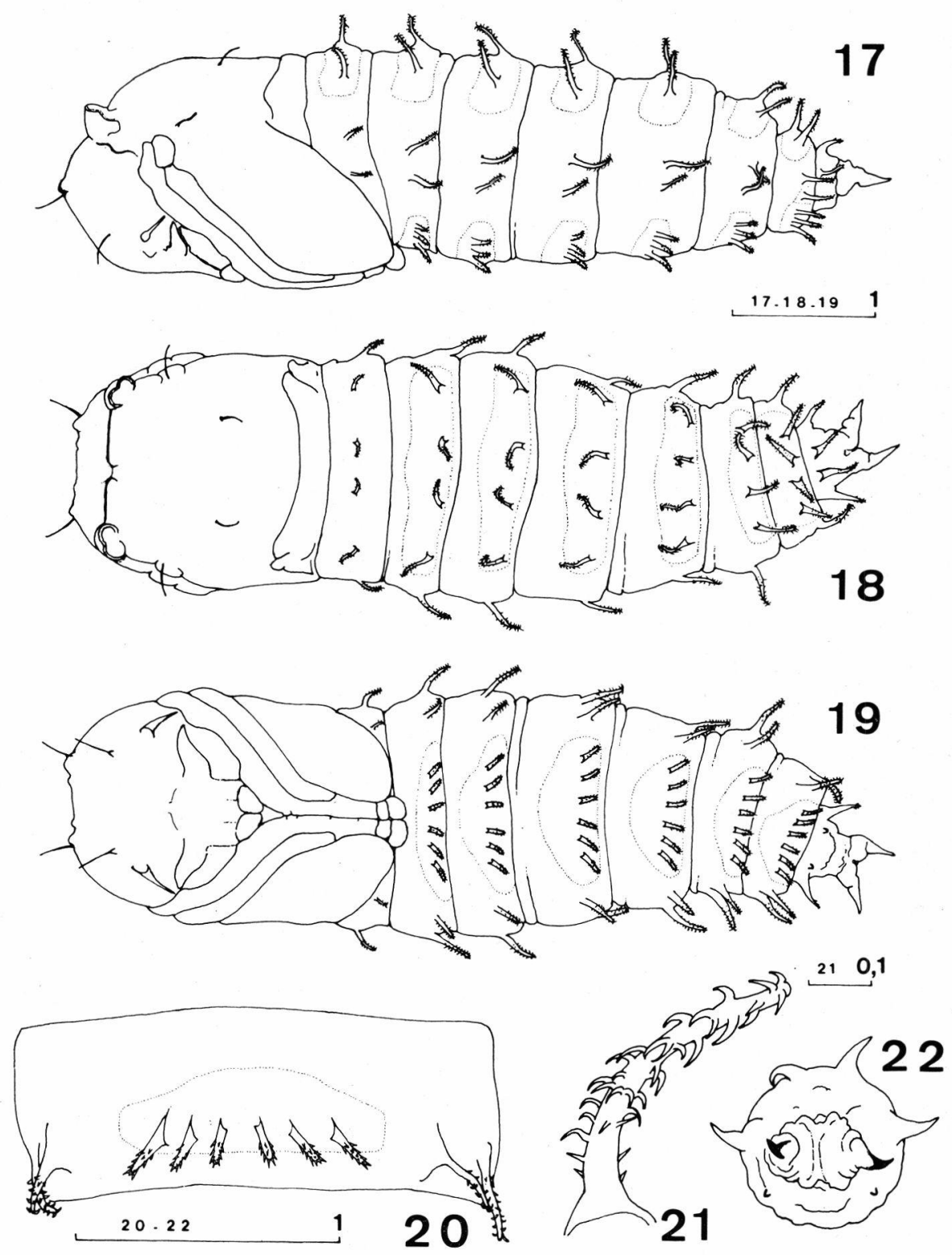

Planche III : fig. 17 à 22 (nymphe d'A. crassipes). Échelle en mm.

FIg. 17 : vue latérale gauche; FIg. 18 : vue dorsale; Fig. 19 : vue ventrale; Fig. $20: 4$ e sternite abdominal; Fig. 21 : prolongement dorso-latéral du 4e tergite; Fig. 22 : segment anal, face postérieure. 
distale atteint le bord de la céphalothèque ; leur angle d'inclinaison par rapport au plan de symétrie est de près de $45^{\circ}$.

\section{Thorax.}

Deux paires de soies bien visibles, l'une à la naissance des ptérothèques, l'autre dorsalement, à peu près au centre du mésothorax. Les ptérothèques sont proportionnellement très développées et recouvrent la totalité du premier sternite. Les pédothèques recouvrent même une partie du second sternite (fig. 19).

\section{AbDomen.}

Chez cette espèce, largeur maximale $d u$ corps au niveau des $3^{\circ}$ et $4^{\mathrm{e}}$ segments abdominaux (prolongements non compris) et non au niveau du mésothorax. Les prolongements dorsaux et latéraux sont beaucoup plus forts que chez les deux autres espèces et sont moins nettement barbelés à la base ( $f i g .21$ ). Les deux prolongements centraux sont de taille quelque peu réduite sur les deux premiers tergites et, au contraire, de taille accrue sur le $7^{\circ}$. Prolongements dorsaux régulièrement espacés (fig. 18), pas de tubercules surnuméraires sur les derniers tergites. Epines sternales (fig. 20) quatre à six fois plus longues que larges, fortes, émoussées à l'extrémité et quelque peu barbelées. Ces épines sont régulièrement espacées.

Les épines du segment anal (fig. 22) ont une morphologie voisine de celle des derniers prolongements dorsaux : leur base est seulement un peu plus large et plus nettement conique. Les deux épines ventrales ont presque disparu et chacune d'elles est remplacée par un très petit tubercule. Les appendices terminaux sont coniques, assez longs et nettement divergents (q).

Matériel examiné : 1 exuvie nymphale.

— Ariège : le Volp à $280 \mathrm{~m}$ ( 1 i. o obtenue par élevage).

\section{TRAVAUX CITES}

Brauns (A.). 1954. - Puppen terricoler Dipterenlarven. 156 p. +54 pl. Göttingen.

Dufour (L.). 1862. - Consultation sur une larve aquatique. Annls Soc. ent. Fr., $4^{\text {e }}$ série, $2: 131-138+1$ pl.

Nagatomi (A.). 1961. - Studies in the aquatic Snipe flies of Japan. Part IV. Descriptions of the pupae (Diptera, Rhagionidae). Mushi, 35 (4) : 29-38,

Pomeisl (E.). 1953. - Studien an Dipterenlarven des Mauerbaches. Wett. Leben, 2 : 165-176.

SÉGUy (E.). 1967. - Dictionnaire des termes techniques d'entomologie élémentaire. 465 p., Lechevalier, Paris.

Thomas (A. G. B.). 1974. - Diptères torrenticoles peu connus : I. - Les Athericidae (larves et imagos) du Sud de la France (Brachycera, Orthorrhapha). Annls Limnol., 10 (1) : 55-84. 